\title{
Caracterização epidemiológica de pacientes submetidos ao transplante de células-tronco hematopoéticas em um centro de referência de Curitiba, Paraná, Brasil, 2011-2015
}

\section{Epidemiological characterization of patients submitted to hematopoietic stem cell transplantation at a reference center in Curitiba, Paraná, Brazil, 2011-2015}

Gisele de Paula e Silva Carneiro Mendes de Souza ${ }^{1}$, Leticia Mara Marca², Milene Zanoni da Silva¹, Doroteia Aparecida Hofelmann¹, Yanna Dantas Rattmann ${ }^{1}$

\section{RESUMO}

Introdução: O transplante de células-tronco hematopoéticas (TCTH) é a única alternativa para o tratamento de algumas doenças. Entretanto, identifica-se escassez de estudos na população brasileira. Objetivo: Caracterizar o perfil dos pacientes submetidos ao TCTH no Hospital de Clínicas da Universidade Federal do Paraná (HC/UFPR), entre 2011 e 2015, com base em variáveis demográficas, diagnóstico, duração da internação, e a taxa de mortalidade na instituição. Métodos: Pesquisa de séries temporais baseada em dados do Sistema de Informações Hospitalares. Avaliou-se a tendência na distribuição das proporções ao longo dos anos por meio do teste de Cochran-Armitage e da regressão binomial negativa. A presença de autocorrelação seriada foi testada pelo teste de Durbin-Watson. Resultados: De 2011-2015 o Paraná foi responsável por 9,2\% dos TCTH realizados no Brasil. O HC/UFPR foi responsável por $46,0 \%$ destes procedimentos realizados no Paraná. Não foram observadas variações significativas na distribuição das variáveis sexo $(p=0,788)$ e número de TCTH $(p=0,213) .59,5 \%$ dos pacientes residiam noPR, 49,4\% tinhamentre 0 e 17 anos, $79,9 \%$ eram brancos, e $63,5 \%$ do sexo masculino. O TCTH alogênico foi o mais realizado (88,5\%). 58,5\% permaneceram internados de 31 a 60 dias (média=37,6 dias). 9,1\% foram a óbito. A anemia aplástica adquirida foi a doença base mais frequente $(31,9 \%)$. Conclusão: $\mathrm{O}$ TCTH é um procedimento de alto custo e complexidade. O estudo e a compreensão dos fatores determinantes para o seu sucesso são de extrema importância para o melhor planejamento, estimativa de risco e elaboração de políticas públicas de saúde.

Palavra-chave: estudos de séries temporais; anemia aplástica; transplante de células-tronco hematopoéticas.

\begin{abstract}
Introduction: Hematopoietic stem cell transplantation (HSCT) is the only alternative for the treatment of some diseases. However, there is a shortage of studies in Brazilian population. Objective: To identify the profile of patients submitted to HSCT at the Hospital de Clínicas da Universidade Federal do Paraná (HC/UFPR), between 2011 and 2015, based on demographic variables, diagnosis, duration of hospitalization and the mortality ration in the institution. Methods: Time Series Studies research based on data from the Hospital Information System. The trend in the distribution of proportions over the years was evaluated through Cochran-Armitage test and negative binomial regression. The presence of serial autocorrelation was tested by the Durbin-Watson test. Results: From 2011-2015 Paraná was responsible for $9.2 \%$ of HSCT performed in Brazil. HC/UFPR accounted for $46.0 \%$ of these procedures performed in Paraná. There were no significant variations in the sex distribution $(p=0.788)$ and number of HSCT $(p=0.213) .59 .5 \%$ of the patients were from PR, $49.4 \%$ were between 0 and 17 years old, $79.9 \%$ were white, and $63.5 \%$ were male. The allogeneic HSCT was the most performed procedure (88.5\%). 58.5\% were hospitalized from 31 to 60 days (mean=37.6 days). 9.1\% died. Acquired aplastic anemia was the most common underlying disease (31.9\%). Conclusion: HSCT is a procedure of high cost and complexity. The study and the understanding of the determinants of its success are of extreme importance for the best planning, risk estimation and elaboration of public health policies.
\end{abstract}

Keywords: time series studies; anemia, aplastic; hematopoietic stem cell transplantation. 


\section{INTRODUÇÃO}

O transplante de células-tronco hematopoéticas (TCTH) tem sido indicado para o tratamento de diversas doenças. Entre elas: doenças que comprometem o funcionamento da medula óssea, como a anemia aplástica, as leucemias e o mieloma múltiplo; imunodeficiências, como a Síndrome de Wiskott-Aldrich; doenças congênitas, como a disceratose congênita; alguns tumores sólidos; e doenças autoimunes, como a esclerose múltipla ${ }^{1}$.

O objetivo do TCTH é destruir as células da medula óssea doente e substituí-las por células normais coletadas de um doador saudável e compatível. Dessa forma, o sistema hematopoiético e imunológico do doente podem ser reconstituidos ${ }^{2}$.

As etapas envolvidas na realização do TCTH podem ser visualizadas na Figura 1, e contemplam: 1) o Pré-Transplante: período no qual o paciente recebe acompanhamento ambulatorial até a internação; 2) o TCTH propriamente dito: inicia-se com a hospitalização integral que, geralmente, ocorre sete dias antes da infusão das células do doador. Os dias precedentes a essa infusão são nomeados como D- e numerados regressivamente. Ao longo desses dias o paciente recebe um esquema terapêutico de quimioterapia e/ou radioterapia que tem por princípio básico destruir a medula doente. O dia da infusão das células do doador é nomeado como D0, e os dias após a infusão são nomeados como D+, numerados progressivamente até a alta hospitalar; 3) o Pós-TCTH: inicia-se após a alta e subdivide-se em imediato, até 100 dias da infusão das células do doador, e tardio, a partir desse marco ${ }^{3}$.

São três as modalidades de TCTH existentes: 1) Alogênico, no qual o paciente recebe as células de outra pessoa, que pode ser algum familiar (doador aparentado) ou não (doador não aparentado); 2) Singênico, no qual o doador é um irmão gêmeo idêntico; 3) Autogênico ou autólogo, no qual são utilizadas as células do próprio paciente 4 .

As células que serão utilizadas no TCTH podem ser obtidas de diferentes fontes: 1) Medula Óssea; 2) Sangue Periférico; 3) Cordão Umbilical; 4) Placenta ${ }^{5,6}$.

Sabe-se que o número de procedimentos de TCTH tem aumentado de modo expressivo, especialmente em países desenvolvidos ${ }^{6}$. Estima-se que 30 a 50 mil transplantes sejam realizados anualmente por todo o mundo. O número absoluto no mundo aumentou de 46.563 em 2006 para 68.146 em 2012, um aumento de $46 \%{ }^{7}$.

Segundo a Associação Brasileira de Transplantes de Órgãos, no período de 2011 a 2015, o Brasil realizou aproximadamente 1975
TCTH por ano ${ }^{8}$. O Paraná se encontra entre os dez estados que mais realizam TCTH no Brasil. Nos anos de 2014 e 2015 o Paraná alcançou a $3^{\circ}$ posição, com 198 transplantes $(9,4 \%)$ e 224 transplantes (10,5\%), respectivamente. Dos transplantes realizados no Paraná no ano de 2014, o Hospital de Clínicas da Universidade Federal do Paraná (HC/UFPR) realizou 37,0\% (74 transplantes) e, no ano de $2015,35,0 \%$ (79 transplantes), confirmando a relevância da instituição no cenário do TCTH estadual e nacional ${ }^{8}$.

O transplante de células-tronco hematopoéticas é classificado pelo Ministério da Saúde como um procedimento de alta complexidade e alto custo. Contudo, identifica-se escassez de indicadores próprios para avaliar os resultados e identificar o estado da arte na população brasileira 9 .

Para o desenvolvimento desses indicadores, a determinação das características epidemiológicas dos pacientes submetidos ao TCTH é de grande importância. As investigações epidemiológicas buscam identificar exaustivamente os determinantes do adoecimento por meio de relações causais analíticas e permitem uma visão mais abrangente sobre a etiologia da doenç ${ }^{10}$.

Além disso, por meio dessa análise obtêm-se informações que podem ser úteis para planejamentos administrativos, pesquisas e evolução clínica dos pacientes. Também permite relacionar o problema de saúde com seus fatores determinantes e identificar os riscos para o acometimento da doença. Dessa forma, fornece elementos para a gestão e assistência de um serviço de saúde ${ }^{11}$.

Historicamente, o HC/UFPR foi inaugurado em 1960 e realiza $100 \%$ dos atendimentos por meio do sistema público de saúde. Em 1979, nessa instituição, um grupo pioneiro de hematologistas deu início aos TCTH no Brasil utilizando a medula óssea de doador como fonte de células ${ }^{12}$

Apesar da importância histórica na assistência ao TCTH do estado do Paraná, o perfil epidemiológico dos pacientes atendidos no HC/UFPR não é conhecido. Dados provenientes de outros estados sugerem um perfil predominante dos pacientes que realizam o TCTH, os quais seriam do sexo masculino, idade entre $40 \mathrm{e}$ 49 anos, e a cor da pele branca ${ }^{13,14}$

No entanto, somente conhecer essas características não é o suficiente para entender o processo saúde-doença envolvido no TCTH. Para melhor compreensão desse processo, além da pesquisa descritiva, este estudo também se propõe a utilizar uma das principais estratégias de investigação para a epidemiologia e para a saúde pública: a análise da distribuição temporal ${ }^{15}$.

\begin{tabular}{|c|c|c|c|c|c|c|c|c|c|c|}
\hline & & & & $\mathrm{D}-\mathrm{N}$ & & Do & & $\mathrm{D}+\mathrm{N}$ & & \\
\hline $\begin{array}{l}\text { Internação na } \\
\text { Unidade de } \\
\text { Transplante }\end{array}$ & $\rightarrow$ & $\begin{array}{l}\text { Tratamento + } \\
\text { Quimioterapia }\end{array}$ & $\rightarrow$ & $\begin{array}{l}\text { Aplasia } \\
\text { medular }\end{array}$ & $\rightarrow$ & Infusão & $\rightarrow$ & $\begin{array}{l}\text { Período de } \\
\text { recuperação }\end{array}$ & $\rightarrow$ & Alta \\
\hline \multicolumn{7}{|c|}{ Transplante } & & \multicolumn{3}{|c|}{ Pós-transplante } \\
\hline
\end{tabular}

Figura 1: Etapas do Transplante Células Tronco Hematopoéticas. 
A análise de séries temporais é uma forma de organizar no tempo as informações quantitativas que podem interferir, favorável ou desfavoravelmente, nos processos em curso e permitir o planejamento ${ }^{15}$.

Sendo assim, esse estudo teve como objetivo identificar o perfil epidemiológico dos pacientes submetidos ao transplante de células-tronco hematopoéticas no Hospital de Clínicas da Universidade Federal do Paraná (HC/UFPR), no período de 2011 a 2015, com base em variáveis demográficas, diagnóstico inicial e duração da internação hospitalar, além de estimar a taxa de mortalidade relacionada ao TCTH na instituição.

\section{MÉTODOS}

Esse estudo corresponde a uma pesquisa de séries temporais, compreendendo informações dos indivíduos submetidos ao transplante de células-tronco hematopoéticas no HC/UFPR, no período de 2011 a 2015.

O HC/UFPR é o maior hospital público do Paraná. Trata-se de um hospital universitário, 100\% Sistema Único de Saúde, com atendimento terciário e de grande porte, dispondo de 406 leitos ativos em $2017^{16}$.

A fonte de dados utilizada para a realização da pesquisa foi secundária e viabilizada por meio do acesso ao Sistema de Informações Hospitalares (SIH), disponível para o controle de todas as intervenções realizadas durante o internamento e/ou atendimento do paciente no HC/UFPR. Foram excluídos do estudo aqueles pacientes que, apesar de terem sido internados para o transplante de células-tronco hematopoéticas, por algum motivo, não o realizaram no período considerado.

As variáveis categóricas analisadas foram: sexo (masculino e feminino), faixa etária (pediátrico de 0 a 17 anos, adulto de 18 a 59 anos e idoso $\geq 60$ anos), cor da pele (amarelo, branco, indígena, preto/pardo), tipo de TCTH realizado (autogênico, alogênico aparentado e alogênico não aparentado), período de internação hospitalar (até 30 dias, de 31 a 60 dias, de 61 a 90 dias, de 91 a 100 dias e $>100$ dias), tipo de liberação dos pacientes (alta e óbito), ocupação (conforme Classificação Brasileira de Ocupações) e doença de base classificada conforme CID 10. As variáveis quantitativas analisadas foram: número de TCTH realizados e número de óbitos ao longo do período estudado.

Os dados obtidos foram digitados em banco eletrônico do Microsoft Excel. Para a elaboração de gráficos foi utilizado o programa Graphpad Prism versão 6. Para análise descritiva dos dados foram empregados: média, valores mínimo e máximo, e porcentagem.

$\mathrm{Na}$ comparação de tendência na distribuição das proporções ao longo dos anos para as variáveis categóricas foi empregado o teste de Cochran-Armitage. Para investigar a tendência na distribuição do número de transplantes, de óbitos, e tipos de transplante no período investigado foi aplicada a regressão binomial negativa. A presença de autocorrelação seriada foi testada por meio do teste de Durbin-Watson, nesse teste, se o valor encontrado estiver próximo de dois indica ausência de autocorrelação seriada.
As análises foram realizadas no programa Stata12, considerando o nível de significância estatística de 0,05.

A pesquisa foi previamente aprovada pelo Comitê de Ética em Pesquisa do Setor de Ciências da Saúde da Universidade Federal do Paraná, conforme Certificado de Apresentação para Apreciação Ética (CAAE) No 51635815.6.0000.0102.

\section{RESULTADOS}

Durante o período estudado, 2011 a 2015, o HC/UFPR atendeu 417 pacientes para o TCTH. Porém, o número de transplantes realizados totalizou 418, pois um dos pacientes realizou dois TCTH em uma mesma internação. O número médio de transplantes no período foi de 83,6, e variou de 74 a 93, em 2014 e 2011, respectivamente (Figura 2). Não houve variação significativa no número de TCTH ao longo dos cinco anos estudados. O valor obtido no teste de Durbin-Watson foi próximo de dois indicando, portanto, ausência de autocorrelação seriada.

A maioria dos pacientes submetidos ao TCTH no período era procedente do Paraná, (59,5\%). Na sequência, surge o estado de Santa Catarina, com 10,1\%, Bahia, com 5,3\%, São Paulo, com $3,4 \%$, Minas Gerais e Distrito Federal, ambos com 2,6\%. Os demais pacientes, $16,5 \%$, foram procedentes de outros estados (RS, AM, GO, PB, PI, CE, AL, PE, MA, RO, PA, ES, RJ, MS, MT e TO).

Ao longo dos 5 anos, observou-se o predomínio do sexo masculino $(63,5 \% \mathrm{p}=0,788)$ quanto ao número de transplantes, mantendo sua estabilidade no período considerado, conforme demonstrado na Tabela 1.

Observou-se que a faixa etária mais prevalente foi a pediátrica (0 a 17 anos) com 49,4\%, seguida da faixa etária adulta (18 a 59 anos) com $47,7 \%$, e da faixa etária idosa ( $\geq 60$ anos) com 2,9\% (Tabela 1). Para a variável ocupação, considerou-se somente os pacientes com idade igual ou superior a 18 anos $(\mathrm{n}=211)$ e foram identificadas falhas de preenchimento, uma vez que constava a ocupação para somente $25,6 \%$ dos pacientes, caracterizando uma limitação do estudo. Entre estes pacientes, as ocupações mais

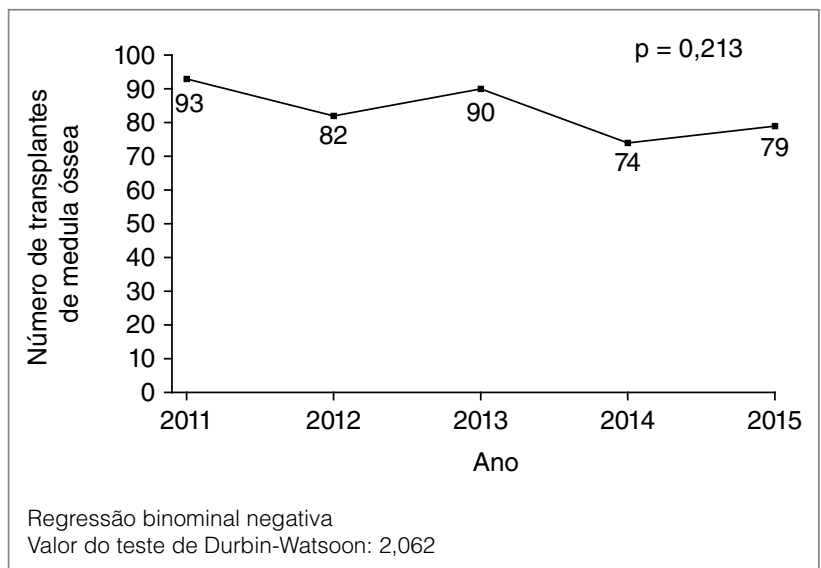

Figura 2: Distribuição do número de transplantes de medula óssea realizados no Hospital de Clínicas - UFPR, 2011-2015. 
frequentes foram estudantes $(4,3 \%)$, trabalhadores de serviços domésticos em geral (2,8\%), aposentados $(2,8 \%)$ e professores $(1,9 \%)$, entre outras ocupações $(13,8 \%)$.

Dos pacientes, $79,9 \%$ foram identificados como brancos, totalizando 333 pacientes (Tabela 1). Como pretos/pardos foram identificados 83 pacientes $(19,9 \%)$. Os indígenas foram representados por 1 único paciente $(0,2 \%)$.

Os pacientes que realizaram o TCTH autogênico totalizaram 11,5\%, o TCTH alogênico aparentado, 44,5\% e o TCTH alogênico não aparentado, 44,0\% (Tabela 1). Quanto à duração da internação hospitalar, 58,5\% (244 pacientes) permaneceram internados de 31 a 60 dias. A média de dias de internação foi de 37,6. A menor duração de internação identificada foi de 7 dias e a maior, de 205 dias (Tabela 1).

Dos 417 pacientes submetidos ao TCTH no HC/UFPR no período avaliado, 379 pacientes $(90,9 \%)$ obtiveram alta médica e 38 pacientes $(9,1 \%)$ foram a óbito (Tabela 1$)$.

A doença diagnosticada de maior prevalência neste estudo, identificada como principal razão para o TCTH foi a Anemia Aplástica Adquirida (AAA). Esta totalizou 134 pacientes $(32,1 \%)$ ao longo dos cinco anos estudados, conforme apresentado na Tabela 2. Em $2^{a}$ posição, encontra-se a Leucemia Linfoblástica
Aguda (LLA), totalizando 66 pacientes (15,8\%). A Leucemia Mielóide Aguda (LMA) aparece em $3^{\circ}$ lugar, somando 40 pacientes $(9,6 \%)$. A Anemia de Fanconi, em $4^{\text {a }}$ posição, com 27 pacientes (6,5\%) (Tabela 2).

As quatro doenças, citadas previamente como as mais frequentes, totalizam $64,0 \%$ dos diagnósticos. Os $36,0 \%$ restantes estão distribuídos em muitos outros diagnósticos, detalhados na Tabela 2.

\section{DISCUSSÃO}

Em 2007, o Serviço de Transplante de Células-Tronco Hematopoéticas (STCTH) do HC/UFPR inaugurou seu Centro de Pesquisa e foi o pioneiro no Brasil a receber o Certificado Internacional do Programa do Doador de Medula, por cumprir $100 \%$ dos requisitos. Em 2009, o STCTH completou 30 anos de existência, tornando-se referência mundial após a realização de quase 2.000 transplantes ${ }^{17}$.

Neste estudo, ao longo do período considerado, 2011 a 2015, o Brasil realizou 9.875 transplantes de células-tronco hematopoéticas. O estado do Paraná realizou 908 transplantes no mesmo período, o que representa 9,2\% dos transplantes realizados no Brasil.

Tabela 1: Distribuição das características dos indivíduos submetidos ao transplante de células-tronco hematopoéticas no Hospital de Clínicas da Universidade Federal do Paraná, 2011-2015

\begin{tabular}{|c|c|c|c|c|c|c|c|}
\hline & $\begin{array}{l}2011 \\
\text { n (\%) }\end{array}$ & $\begin{array}{l}2012 \\
\text { n (\%) }\end{array}$ & $\begin{array}{l}2013 \\
\text { n (\%) }\end{array}$ & $\begin{array}{l}2014 \\
\text { n (\%) }\end{array}$ & $\begin{array}{l}2015 \\
\text { n (\%) }\end{array}$ & $\begin{array}{l}\text { Total } \\
\text { n (\%) }\end{array}$ & $\mathbf{p}$ \\
\hline \multicolumn{8}{|l|}{ Sexo } \\
\hline Masculino* & $58(62,4)$ & $54(66,7)$ & $59(65,6)$ & $44(59,5)$ & $50(63,3)$ & $265(63,5)$ & \\
\hline Feminino & $35(37,6)$ & $27(33,3)$ & $31(34,4)$ & $30(40,5)$ & $29(36,7)$ & $152(36,5)$ & $0,788^{\circ}$ \\
\hline \multicolumn{8}{|l|}{ Faixa etária } \\
\hline Pediátricos* (0 a 17 anos) & $46(49,5)$ & $48(59,3)$ & $41(45,6)$ & $33(44,6)$ & $38(48,1)$ & $206(49,4)$ & \\
\hline Adultos (18 a 59 anos) & $46(49,4)$ & $32(39,5)$ & $44(48,9)$ & $37(50,0)$ & $40(50,6)$ & $199(47,7)$ & $0,417^{\bullet}$ \\
\hline Idosos ( $\geq 60$ anos) & $1(1,1)$ & $1(1,2)$ & $5(5,5)$ & $4(5,4)$ & $1(1,3)$ & $12(2,9)$ & $0,335^{\circ}$ \\
\hline \multicolumn{8}{|l|}{ Raça } \\
\hline Amarelos & - & - & - & - & - & - & - \\
\hline Brancos $^{*}$ & $85(91,4)$ & $61(75,3)$ & $64(71,1)$ & $59(79,7)$ & $64(81,0)$ & $333(79,9)$ & \\
\hline Indígenas & - & - & $1(1,1)$ & - & - & $1(0,2)$ & $\times$ \\
\hline Pretos e Pardos** & $8(8,6)$ & $20(24,7)$ & $25(27,8)$ & $15(20,3)$ & $15(19,0)$ & $83(19,9)$ & $0,168^{\circ}$ \\
\hline \multicolumn{8}{|l|}{ Tipos de transplante $(n=418)$} \\
\hline Autogênico & $15(16,2)$ & $7(8,5)$ & $11(12,2)$ & $7(9,5)$ & $8(10,1)$ & $48(11,5)$ & $\times$ \\
\hline Alogênico aparentado* & $39(41,9)$ & $39(47,6)$ & $39(43,3)$ & $30(40,5)$ & $39(49,4)$ & $186(44,5)$ & \\
\hline Alogênico não aparentado & $39(41,9)$ & $36(43,9)$ & $40(44,5)$ & $37(50,0)$ & $32(40,5)$ & $184(44,0)$ & 0,879 • \\
\hline \multicolumn{8}{|l|}{ Período de internação } \\
\hline Até 30 dias $^{*}$ & $33(35,5)$ & $20(24,7)$ & $27(30,0)$ & $28(37,8)$ & $31(39,2)$ & $139(33,3)$ & \\
\hline 31 a 60 dias & $54(58,0)$ & $54(66,7)$ & $55(61,1)$ & $39(52,7)$ & $42(53,2)$ & $244(58,5)$ & $0,213^{\circ}$ \\
\hline 61 a 90 dias & $4(4,3)$ & $2(2,5)$ & $5(5,6)$ & $4(5,4)$ & $3(3,8)$ & $18(4,3)$ & $0,938^{\circ}$ \\
\hline 91 a 100 dias & $1(1,1)$ & $1(1,2)$ & $3(3,3)$ & $1(1,4)$ & - & $6(1,5)$ & $0,552^{\bullet}$ \\
\hline$>100$ dias & $1(1,1)$ & $4(4,9)$ & - & $2(2,7)$ & $3(3,8)$ & $10(2,4)$ & $0,724^{\circ}$ \\
\hline \multicolumn{8}{|l|}{ Liberação dos pacientes } \\
\hline Alta médica* & $82(88,2)$ & $72(88,9)$ & $83(92,2)$ & $69(93,2)$ & $73(92,4)$ & $379(0,9)$ & \\
\hline Óbito*** & $11(11,8)$ & $9(11,1)$ & $7(7,8)$ & $5(6,8)$ & $6(7,6)$ & $38(9,1)$ & $0,195^{\circ}$ \\
\hline
\end{tabular}

*Classe utilizada como referência para comparação nos testes estatísticos.

${ }^{\star *} \mathrm{O}$ sistema utiliza a letra P para identificar Pretos e Pardos:

*** Óbito exclusivamente durante a internação.

- Teste estatístico Cochran-Armitage.

× Análise não convergiu número reduzido ou nulo de observações. 
Dos 908 transplantes realizados no estado, o HC/UFPR contribuiu com 418 transplantes, 4,2\% dos TCTH realizados no Brasil e 46,0\% dos TCTH realizados no Paraná. Estes números indicam a relevância do STCTH do HC/UFPR para o estado do Paraná e para o Brasil ${ }^{8}$.

Nos transplantes de células-tronco hematopoéticas, o predimínio de pacientes do sexo masculino é comumente constatado em vários estudos, a exemplo dos estudos de Azevedo ${ }^{13}$, Silva e Pasquini ${ }^{18}$, nos quais o sexo masculino representou $52,9 \%$ e $64,0 \%$ dos transplantados, respectivamente. Dado o exposto, frequentemente aventa-se a hipótese de que os homens são mais acometidos por neoplasias hematológicas, possivelmente em decorrência da maior exposição a agentes físicos, como radiação ionizante, e elementos químicos, como o benzeno ${ }^{14}$.
Na América Latina, a média de idade dos pacientes submetidos ao TCTH alogênico é 30 anos, e dos que passam pelo TCTH autogênico é 45 anos $^{19}$. Entretanto, a faixa etária de maior prevalência no atual estudo foi de 0 a 17 anos, diferente do especificado na literatura científica. Provavelmente, isto se deve ao fato de que 32,1\% dos pacientes da amostra apresentaram AAA como diagnóstico. Para essa doença o TCTH alogênico, aparentado ou não aparentado, está indicado ${ }^{20}$. A maior incidência desta doença ocorre em indivíduos entre 10 e 25 anos ou em maiores de 60 anos, sem distinção de sexo $^{20}$. Neste estudo, $52,6 \%$ dos pacientes com AAA pertencem à faixa etária entre 10 e 25 anos. Nenhum paciente acima de 60 anos foi identificado, justificando o baixo número de idosos na amostra.

Ademais, o reduzido número de idosos pode ser justificado pela gravidade e frequência das complicações pós-transplante neste grupo ${ }^{21}$.

Tabela 2: Doença primária diagnosticada nos pacientes submetidos ao transplante de células-tronco hematopoéticas no Hospital de Clínicas da Universidade Federal do Paraná no período de 2011 a 2015

\begin{tabular}{|c|c|c|c|c|c|c|c|}
\hline \multirow{2}{*}{$\begin{array}{l}\text { CID-10 } \\
\text { C00-D48 }\end{array}$} & \multirow{2}{*}{\multicolumn{2}{|c|}{$\begin{array}{l}\text { Denominação da classe } \\
\text { Neoplasias (tumores) }\end{array}$}} & \multicolumn{5}{|c|}{$\mathrm{N}^{\circ}$ absoluto de pacientes por ano } \\
\hline & & & 2011 & 2012 & 2013 & 2014 & 2015 \\
\hline \multirow{4}{*}{$\begin{array}{l}\text { Grupo entre } \\
\text { C81 e C96 }\end{array}$} & \multirow{4}{*}{$\begin{array}{l}\text { Neoplasias [tumores] malignas(os), } \\
\text { declaradas ou presumidas como primárias, } \\
\text { dos tecidos linfático, hematopoético e } \\
\text { tecidos correlatos }\end{array}$} & Leucemia Linfoblástica aguda (C91.0) & 15 & 19 & 13 & 10 & 9 \\
\hline & & Leucemia Mielóide Aguda (C92.0) & 14 & 6 & 6 & 5 & 9 \\
\hline & & Leucemia Mielóide Crônica (C92.1) & 3 & 3 & 7 & 1 & 5 \\
\hline & & Outras & 16 & 11 & 13 & 8 & 10 \\
\hline & Total & & 48 & 39 & 39 & 24 & 33 \\
\hline \multirow{3}{*}{$\begin{array}{l}\text { Grupo entre } \\
\text { D37 e D48 }\end{array}$} & \multirow{2}{*}{$\begin{array}{l}\text { Neoplasias [tumores] de comportamento } \\
\text { incerto ou desconhecido }\end{array}$} & Sindrome mielodisplásica (D46.9) & 2 & 5 & 1 & 6 & 2 \\
\hline & & Síndrome de POEMS (D47.7) & - & - & 1 & - & - \\
\hline & \multicolumn{2}{|l|}{ Total } & 2 & 5 & 2 & 6 & 2 \\
\hline D50-D89 & \multicolumn{7}{|c|}{ Doenças do sangue e dos órgãos hematopoéticos e alguns transtornos imunitários. } \\
\hline $\begin{array}{l}\text { Grupo entre } \\
\text { D55 e D59 }\end{array}$ & Anemias hemolíticas & Anemia Hemolítica auto-imune (D59) & - & - & - & - & 1 \\
\hline \multicolumn{3}{|c|}{ Total } & - & - & - & - & 1 \\
\hline \multirow{3}{*}{$\begin{array}{l}\text { Grupo entre } \\
\text { D60 e D64 }\end{array}$} & \multirow{3}{*}{ Anemias aplásticas e outras anemias } & Anemia Aplástica adquirida (D61) & 24 & 22 & 34 & 30 & 24 \\
\hline & & Anemia de Fanconi (D61.0) & 5 & 3 & 5 & 5 & 9 \\
\hline & & Anemia Disertropoetica congenita (D64) & 1 & - & - & - & - \\
\hline \multirow{4}{*}{$\begin{array}{l}\text { Grupo entre } \\
\text { D70 e D77 }\end{array}$} & \multirow{4}{*}{$\begin{array}{c}\text { Outras doenças do sangue e dos órgãos } \\
\text { hematopoéticos }\end{array}$} & & 30 & 25 & 39 & 35 & 33 \\
\hline & & Sindrome de Kostmann (D70) & 2 & - & - & - & - \\
\hline & & Doença Granulomatosa Crônica (D71) & - & 1 & - & - & - \\
\hline & & Linfohistiocitose Hemofagocítica (D76.1) & 1 & - & - & - & - \\
\hline \multicolumn{3}{|c|}{ Total } & 3 & 1 & - & - & - \\
\hline \multirow{3}{*}{$\begin{array}{l}\text { Grupo entre } \\
\text { D80 e D89 }\end{array}$} & \multirow{3}{*}{$\begin{array}{l}\text { Alguns transtornos que comprometem o } \\
\text { mecanismo imunitário }\end{array}$} & $\begin{array}{l}\text { Síndrome de imunodeficiência combinada } \\
\text { (D81) }\end{array}$ & - & 2 & - & 1 & 2 \\
\hline & & Síndrome de Wiskott -Aldrich (D82.0) & 2 & 6 & 5 & 3 & 4 \\
\hline & & Outras & 1 & - & - & 2 & 2 \\
\hline & \multicolumn{2}{|l|}{ Total } & 3 & 8 & 5 & 6 & 8 \\
\hline E00-E90 & \multicolumn{7}{|c|}{ Doenças endócrinas, nutricionais e metabólicas } \\
\hline \multirow{4}{*}{$\begin{array}{l}\text { Grupo entre } \\
\text { E70 e E90 }\end{array}$} & \multirow{3}{*}{ Distúrbios metabólicos } & Síndrome de Chediak-Higashi (E70.3) & - & - & - & 1 & - \\
\hline & & Adrenoleucodistrofia ligada ao X (E71.3) & 1 & 1 & 2 & - & - \\
\hline & & Mucopolissacaridose (E76) & 1 & 2 & - & - & - \\
\hline & Total & & 2 & 3 & 2 & 1 & - \\
\hline G00-G99 & \multicolumn{7}{|c|}{ Doenças do sistema nervoso } \\
\hline $\begin{array}{l}\text { Grupo entre } \\
\text { G35 e G37 }\end{array}$ & $\begin{array}{c}\text { Doenças desmielinizantes do sistema } \\
\text { nervoso central }\end{array}$ & Esclerose múltipla (G35) & 5 & - & 1 & - & - \\
\hline \multicolumn{3}{|c|}{ Total } & 5 & - & 1 & - & - \\
\hline Q00-Q99 & \multicolumn{7}{|c|}{ Malformações congênitas, deformidades e anomalias cromossômicas } \\
\hline $\begin{array}{l}\text { Grupo entre } \\
\text { Q80 e Q89 }\end{array}$ & Outras malformações congênitas & Disceratose congênita (Q82.8) & - & 1 & 2 & 2 & 2 \\
\hline \multicolumn{3}{|c|}{ Total } & - & 1 & 2 & 2 & 2 \\
\hline
\end{tabular}


O transplante de células-tronco hematopoéticas em idosos doentes pode aumentar o risco de toxicidades específicas, tais como toxicidades cardíacas e mucosite, além de apresentar menor eficácia $^{22}$. Segundo Federmann et al. ${ }^{23}$ a incidência da doença do enxerto contra o hospedeiro aumenta conforme o acréscimo da idade e pode prejudicar o resultado do TCTH e a qualidade de vida do paciente. A doença do enxerto contra o hospedeiro é uma complicação comum do TCTH e tem um impacto significativo na morbidade e mortalidade precoce ${ }^{24}$. Esta doença é desencadeada por uma resposta imunológica aos antígenos naturais ou aos expressos devido ao dano tecidual gerado pela doença de base ou ainda pelo condicionamento ${ }^{25}$.

Outro fator que limita a realização do TCTH em idosos é a toxicidade da quimioterapia e a presença de comorbidades, que exercem influência negativa sobre o resultado do transplante ${ }^{23}$. As comorbidades e o estado do paciente idoso são provavelmente melhores preditores de toxicidade do que a idade cronológica ${ }^{22}$.

A cor de pele branca, proporcionalmente maior entre os indivíduos que realizam o TCTH, não parece ter relação com a evolução clínica das doenças diagnosticadas nestes pacientes ${ }^{26}$, uma vez que a indicação para o transplante independe da cor de pele. Outros fatores são considerados para compreensão do baixo número de TCTH em indivíduos de cores de pele que não a branca. Entre eles parecem interferir os fatores socioeconômicos, que influenciam no acesso a bens e serviços, incluindo os ocupados por médicos, mais disponíveis para a população branca em relação às demais ${ }^{26}$.

As desigualdades de acesso aos serviços médicos parecem sofrer influência do racismo, que opera não reconhecendo as necessidades particulares das minorias nas ações de promoção, prevenção e assistência a doenças e à reabilitação. Confirma-se, portanto, a vulnerabilidade, a acessibilidade diferenciada e dificultada a equipamentos sociais, que culminam no tratamento iníquo segundo cor de pele, comprometendo a equidade e reproduzindo desigualdade ${ }^{27}$.

Neste estudo, os pacientes com cor de pele branca foram maioria, totalizando $79,9 \%$ dos atendidos no HC/UFPR, no período de 2011 a 2015 para realização do TCTH. Outras cores de pele representaram somente $20,1 \%$, evidenciando que ainda existe iniquidade no acesso à saúde no Brasil.

Em relação aos aspectos biológicos, os bancos de medula óssea do mundo todo, apesar do grande número de doadores de medula óssea registrados, enfrentam dificuldade na identificação de doadores para pacientes pertencentes às minorias raciais. Isso acontece devido ao menor número de doadores potenciais entre as minorias raciais ${ }^{28}$.

O antígeno leucocitário humano (HLA) determina a histocompatibilidade da medula óssea entre doador e receptor ${ }^{29}$ e é herdado dos ancestrais sendo, portanto, mais facilmente compartilhado entre indivíduos da mesma cor de pele ${ }^{30}$. Algumas especificidades do HLA são características de uma população, mas podem estar ausentes em outra. Por exemplo, o tipo HLA A-43 é encontrado quase exclusivamente em negros e o HLA B-46 em chineses ${ }^{31}$.
Portanto, para aumentar o número de TCTH nas minorias raciais é necessário que essa população tenha maior conhecimento sobre o tema e que seja incluída no Cadastro Nacional de Medula Óssea ${ }^{32}$.

O baixo número de TCTH autogênicos observado na instituição, no período considerado, pode ser explicado pelo fato de que apenas $22 \%$ dos pacientes apresentavam diagnóstico de doenças passíveis de tratamento/cura com o TCTH autogênico. Em contrapartida, o alto número de TCTH alogênico realizado deve-se, principalmente, ao fato de que a doença primária de maior prevalência foi a anemia aplástica adquirida $(32,1 \%)$, para a qual o TCTH alogênico é o indicado, aparentado ou não ${ }^{24}$.

O período de hospitalização para a realização do TCTH normalmente é longo, em torno de quatro a seis semanas. Neste período ocorre a pega medular, que consiste na ocupação dos espaços vazios pelas células-tronco hematopoéticas, repovoando a medula óssea e restabelecendo a hematopoese. A pega medular é bem sucedida quando a contagem dos neutrófilos supera $500 / \mathrm{mm}^{3}$ e se mantém por dois dias seguidos ${ }^{33}$.

Segundo o Sistema de Gerenciamento da Tabela de Procedimentos, Medicamentos e Órteses, Próteses e Materiais Especiais do SUS (SIGTAP) ${ }^{34}$, o tempo médio de internação de pacientes que realizam o transplante alogênico aparentado é de 29,67 dias, enquanto o transplante alogênico não aparentado requer 33,33 dias de internação. Portanto, o período de internação encontrado neste estudo, cuja média correspondeu a 37,58 dias, encontra-se próximo à média de dias apresentada pelo SIGTAP, e em conformidade com o relatado no estudo de Andrade et al. ${ }^{33}$.

Neste estudo, a taxa de mortalidade relacionada ao TCTH correspondeu a $9,1 \%$. Vale ressaltar que este valor se refere exclusivamente ao período de internação para a realização do transplante, tendo como desfecho a alta ou o óbito. O coeficiente de mortalidade encontrado nesse estudo não pode, portanto, ser utilizado para determinar o sucesso ou o insucesso do TCTH, pois recomenda-se o seguimento dos pacientes ao longo dos cinco anos seguintes ao transplante. Estabeleceu-se esse prazo em razão das neoplasias possuírem uma taxa de sobrevida média de cinco anos, durante os quais a doença pode ou não desaparecer completamente ${ }^{35}$. A taxa encontrada neste estudo foi próxima às encontradas em estudos semelhantes, como Santos ${ }^{36}$ e Cabral et al. ${ }^{14}$, que obtiveram, respectivamente, os valores de $9,5 \%$ e $14,0 \%$.

A anemia aplástica adquirida teve prevalência elevada no atual estudo quando comparada a dados encontrados na literatura. Eckrich e Pasquini ${ }^{19}$ e Cabral et al. ${ }^{14}$ obtiveram as prevalências $19 \%$ e inferior a $10 \%$, respectivamente. A prevalência elevada pode ser explicada por ser o HC/UFPR referência nacional para o diagnóstico e tratamento de pacientes com AAA. Curiosamente, as recomendações do protocolo de condicionamentos para o TCTH na AAA foram estabelecidas com base nas experiências do HC/UFPR e do Hospital de clínicas de São Paulo ${ }^{37}$.

$\mathrm{Na}$ condição de hospital de referência para anemia aplástica, anemia de Fanconi e imunodeficiências ${ }^{17}$, o HC/UFPR dispõe de dados 
que podem diferir de outros centros especializados em TCTH. Portanto, as indicações para a realização do TCTH são peculiares e podem não refletir a incidência das doenças citadas. Como exemplo, na Europa, em 2014, os centros de TCTH tiveram como principais doenças de base pré-TCTH as leucemias, síndrome mielodisplásica, linfoma de Hodking e não Hodking. A AAA foi a $23^{\circ}$ doença com maior número de indicações para TCTH naquele $\mathrm{ano}^{38}$.

Desta forma, observa-se que a alta frequência de diagnósticos de doenças raras com indicação para o TCTH reflete a especialização do HC/UFPR, a primeira instituição a realizar este procedimento no Brasil utilizando a medula óssea de doador como fonte de células.

Este estudo contribui com indicadores próprios provenientes do HC/UFPR e reafirma a relevância da instituição no diagnóstico e tratamento de pacientes com anemia aplástica, anemia de Fanconi e imunodeficiências. O TCTH é um procedimento de alto custo e complexidade que representa a possibilidade de tratamento ou cura para estas, entre muitas outras doenças. Portanto, o conhecimento do perfil epidemiológico dos pacientes e a compreensão dos fatores determinantes para o sucesso do procedimento são de extrema relevância, uma vez que podem contribuir com melhor planejamento, para a estimativa de risco e ainda para a elaboração de políticas públicas de saúde. Estas informações adquirem maior apelo sobretudo quando se considera que o HC/UFPR é um hospital público, sujeito, portanto, aos princípios da universalidade, equidade e integralidade do subfinanciado Sistema Único de Saúde.

\section{AGRADECIMENTOS}

Os autores agradecem ao serviço de transplante de células-tronco hematopoéticas do HC/UFPR.

\section{REFERÊNCIAS}

1. Wingard JR, Vogelsang GB, Deeg HJ. Stem call transplantation: supportive care and long-term complications. Hematology Am Soc Hematol Educ Program. 2002;2002(1):422-44 http://dx.doi.org/10.1182/asheducation-2002.1.422

2. Oliveira-Cardoso EA, Mastropietro AP, Voltarelli JC, Santos MA. Qualidade de vida de sobreviventes do transplante de células-tronco hematopoéticas: um estudo prospectivo. Psic Teor Pesq. 2009;25(4):621-8 http://dx.doi.org/10.1590/S0102-37722009000400018

3. Ortega ETT, Lima DH, Veran MP, Kojo TK, Neves MI. Compêndio de enfermagem em transplante de células tronco hematopoéticas: rotinas e procedimentos em cuidados essenciais e em complicações. Curitiba: Editora Maio, 2004; p.435.

4. Castro JR CG. Análise Clínica e Epidemiológica do Transplante de Medula Óssea no serviço de oncologia pediátrica do Hospital de Clínicas de Porto Alegre. Dissertação (Mestrado) Faculdade de Medicina, Universidade Federal do Rio Grande do Sul. Porto Alegre: 2002; p.161.

5. Brasil. Ministério da Saúde. Portaria MS no 931, de 02 de maio de 2006. Aprova o Regulamento técnico para transplante de célulastronco hematopoéticas. Diário Oficial da União. 2006;48-52.

6. Hodgkinson KM, Kiernan J, Shih AW, Solh Z, Sheffield WP, Pineault N. Intersecting Worlds of Transfusion and Transplantation Medicine: An International Symposium Organized by the Canadian Blood Services Centre for Innovation. Transfus Med Rev. 2017;31(3):183-92. http://dx.doi.org/10.1016/j.tmrv.2017.03.001

7. Niederwieser D, Baldomero H, Szer J, Gratwohl MAM, Aljurf M, Atsuta $\mathrm{Y}$, et al. Hematopoietic stem cell transplantation activity worldwide in 2012 and a SWOT analysis of the Worldwide Network for Blood and Marrow Transplantation Group including the global survey. Bone Marrow Transplant. 2016;51(6):778-85. http://dx.doi.org/10.1038/bmt.2016.18

8. Associação Brasileira de Transplante de Órgãos (ABTO). Dimensionamento dos transplantes no Brasil e em cada estado (2008-2015). Registro Bras Transp. 2015;21(4):6-81.

9. Sousa, AM. Fatores prognósticos para sobrevivência após transplante de células-tronco hematopoéticas em portadores de anemia aplástica. Tese (Mestrado) - Escola Nacional de Saúde Pública Sérgio Arouca. Rio de Janeiro: 2012; p.113.
10. Yanagi Y, Assunção JV, Barrozo LV. The impact of atmospheric particulate matter on cancer incidence and mortality in the city of São Paulo, Brazil. Cad Saúde Pública. 2012;28(9):1737-48. http://dx.doi.org/10.1590/S0102-311X2012000900012

11. Andrade CT, Magedanz AMPCB, Escobosa DM, Tomaz WM, Santinho CS, Lopes TO, et al. A importância de uma base de dados na gestão de serviços de saúde. Einsten. 2012;10(3);360-5. http://dx.doi.org/10.1590/S1679-45082012000300018

12. Corgozinho MM, Gomes JRAA, Garrafa V. Transplantes de medula óssea no Brasil: Dimensão Bioética. Rev Latinoam Bioet. 2012;12(1):36-45.

13. Azevedo IC. Perfil clínico, epidemiológico e sobrevida dos transplantados com celúlas-tronco hematopoéticas em um serviço de referência no Rio Grande do Norte. Tese (Mestrado) - Universidade Federal do Rio Grande do Norte. Natal: 2016; p.77.

14. Cabral DCP, Silva RCP, Lacerda ACT, Diniz JMT, Guedes DBB. Análise dos transplantes de medula óssea realizados em pernambuco no período de 2011 a 2012. Rev Bras Med. 2014;71(Esp. 1):2-10.

15. Antunes JLF, Cardoso MRA. Uso da análise de séries temporais em estudos epidemiológicos. Epidemiol Serv Saúde. 2015;24(3):565-76.

http://dx.doi.org/10.5123/S1679-49742015000300024

16. Empresa Brasileira de Serviços Hospitalares (EBSERH). HC em números. Disponível em: http://www.ebserh.gov.br/web/chc-ufpr/ hcnumeros. Acesso em: 03 Maio 2017.

17. Hospital de Clínicas, Universidade Federal do Paraná (UFPR). 50 anos. Rev Hospital. 2011;(Ed esp.):10-20.

18. Silva LCL, Pasquini R. Análise da rejeição nos pacientes transplantados por anemia aplástica severa condicionados com ciclofosfamida ou a associação desta ao bussulfano. Rev Bras Hematol Hemoter. 2005;27(1):5-11. http://dx.doi.org/10.5123/S1679-49742015000300024

19. Eckrich M, Pasquini M. Hematopoietic cell transplantation in Latin America. Hematology. 2012;17(Supl. 1):189-92. http://dx.doi.org/10.1179/102453312X13336169157059 
20. Brasil. Ministério da Saúde. Secretaria de Atenção à Saúde. Portaria SAS/MS n 1300, de 21 de novembro de 2013. Aprova o protocolo clínico e diretrizes terapêuticas da Anemia Aplástica Adquirida. Diário Oficial União. 2013;66-68.

21. Tabak DG, Pereira SCM, Nogueira MD. Transplante de célula-tronco hematopoética para síndrome mielodisplásica. Rev Bras Hematol Hemoter. 2010;32(Supl. 1):66-70. http://dx.doi.org/10.1590/S1516-84842010005000016

22. Wildes TM, Stirewalt DL, Medeiros B, Hurria A. Hematopoietic stem cell transplantation for hematologic malignancies in older adults: geriatric principles in the transplant clinic. J Natl Compr Cancer Netw. 2014;12(1):128-36.

23. Federmann B, Faul C, Meisner C, Vogel W, Kanz L, Bethge WA. Influence of age on outcome after allogeneic hematopoietic cell transplantation: a single centar study in patients aged $\geq 60$. Bone Marrow Transpl. 2015;50(3):427-31. http://dx.doi.org/10.1038/bmt.2014.292

24. Jagasia M, Arora M, Flowers M, Chao NJ, McCarthy PL, Cutler CS, et al. Risk factors for acute GVHD and survival after hematopoietic cell transplantation. Blood. 2012:119(1):296-307. http://dx.doi.org/10.1182/blood-2011-06-364265

25. Azevedo W. Doença enxerto versus hospedeiro aguda A-GVHD. Rev Bras Hematol Hemoter. 2010;32(Supl. 1):16-21. http://dx.doi.org/10.1590/S1516-84842010005000060

26. Joshua TV, Rizzo JD, Zhang MJ, Hari PN, Kurian S, Pasquini M, et al. Access to Hematopoietic Stem Cell Transplantation Effect of Race and Gender. Cancer. 2010;116(14):3469-76. http://dx.doi.org/10.1002/cncr.25297

27. Volochko A, Vidal N de P. Desigualdades raciais na saúde: mortalidade nas regiões de saúde paulistas, 2005. Bol Inst Saúde. 2010;12(2):143-53

28. Switzer GE, Bruce JG, Myaskovsky L, DiMartini A, Shellmer D, Confer DL, et al. Race and ethnicity in decisions about unrelated hematopoietic stem cell donation. Blood. 2013;121(8):1469-76. http://dx.doi.org/10.1182/blood-2012-06-437343

29. Little MT, Storb R. History of haematopoietic stem-cell transplantation. Nat Rev Cancer. 2002;2(3):231-8. http://dx.doi.org/10.1038/nrc748
30. Bicalho MG. HLA e minorias étnicas: quando menos significa muito. Rev Bras Hematol Hemoter. 2010;32(2):101 http://dx.doi.org/10.1590/S1516-84842010000200006

31. Ruiz TM, Costa SM, Ribas F, Luz PR, Lima SS, Bicalho MG. Human leukocyte antigen allelic groups and haplotypes in a brazilian sample of volunteer donors for bone marrow transplant in Curitiba, Paraná, Brazil. Transplant Proc. 2005;37(5):2293-6. http://dx.doi.org/10.1016/j.transproceed.2005.03.036

32. Watanabe AM, Omotto CA, Colli LD, Hayashi VMH. Percepção da comunidade nipo-brasileira residente em Curitiba sobre o cadastro de medula óssea. Rev Bras Hematol Hemoter. 2008;32(2):136-140.

http://dx.doi.org/10.1590/S1516-84842010005000042

33. Andrade AM, Castro EAB, Soares TC, Santos KB. Vivências de adultos submetidos ao transplante de células-tronco hematopoéticas autólogo. Ciência Cuid Saúde. 2012;11(2):267-74. http://dx.doi.org/10.4025/cienccuidsaude.v11i2.15180

34. Brasil. Ministério da Saúde. Sistema de Gerenciamento da Tabela de Procedimentos, Medicamentos e Órteses, Próteses e Materiais especiais do SUS. Disponível em: http://sigtap.datasus.gov.br/ tabela-unificada/app/sec/inicio.jsp. Acesso em: 25 jan. 2017.

35. Campos LG, Paz AA, Silla LMR, Daudt LE. Sobrevida de pacientes submetidos a transplante alogênico de medula óssea. Rev HCPA. 2009;29(2):127-32

36. Santos KBS. Infecções no transplante de células-tronco hematopoéticas no hospital universitário da UFJF: a incidência dos principais microorganismos e os fatores de risco associado. Tese (Mestrado) - Universidade Federal de Juiz de Fora. Juiz de Fora: 2010.

37. Medeiros LA, Pasquini R. Anemia aplástica adquirida e anemia de Fanconi - Diretrizes Brasileiras em transplante de células-tronco hematopoéticas. Rev Bras Hematol Hemoter 2010:32(Supl. 1):40-5.

http://dx.doi.org/10.1590/S1516-84842010005000064

38. Passweg JR, Baldomero H, Bader P, Bonini C, Cesaro S, Dreger $\mathrm{P}$, et al. Hematopoietic stem cell transplantation in Europe 2014 more than 40000 transplants annually. Bone Marrow Transplant. 2016;51(6):786-92

http://dx.doi.org/10.1038/bmt.2016.20 